\title{
Noninvasive continuous cardiac output and cerebral perfusion monitoring in term infants with neonatal encephalopathy: assessment of feasibility and reliability
}

\author{
Eva Forman ${ }^{1}$, Colm R. Breatnach ${ }^{1}$, Stephanie Ryan ${ }^{2}$, Jana Semberova ${ }^{3}$, Jan Miletin ${ }^{3}$, Adrienne Foran ${ }^{1}$ and Afif EL-Khuffash ${ }^{4}$
}

BACKGROUND: Noninvasive hemodynamic monitoring of infants with neonatal encephalopathy (NE) undergoing therapeutic hypothermia (TH) would be a potentially useful clinical tool. We aimed to assess the feasibility and reliability of noninvasive cardiac output monitoring (NICOM) and nearinfrared spectroscopy (NIRS) in this cohort.

METHODS: NICOM and NIRS were commenced to measure cardiac output (CO), systemic vascular resistance (SVR), blood pressure (BP), and cerebral regional oxygen saturations $\left(\mathrm{SctO}_{2}\right)$ during $\mathrm{TH}$ and rewarming. NICOM measures of $\mathrm{CO}$ were also compared with simultaneous echocardiography-derived CO (echo-CO).

RESULTS: Twenty infants with a median gestation of 40 weeks were enrolled. There was a strong correlation between NICOM- and echo-CO $\left(r^{2}=0.79, P<0.001\right)$. NICOM-CO was systematically lower than echo-CO with a bias of 27\% (limits of agreement 3-51\%). NICOM illustrated lower CO during TH, which increased during rewarming. $\mathrm{SctO}_{2}$ increased over the first $30 \mathrm{~h}$ of $\mathrm{TH}$ and stayed high for the remainder of the study. There was a rise in SVR over the first $30 \mathrm{~h}$ of $\mathrm{TH}$ and a decrease during rewarming (all $P<0.05$ ).

CONCLUSIONS: Noninvasive hemodynamic assessment of infants with NE is feasible and illustrates potentially important changes. Larger studies are needed to assess the clinical applicability of those methods in this cohort.

$T$ erm infants sustaining a hypoxic-ischemic perinatal insult resulting in neonatal encephalopathy (NE) are considered for therapeutic hypothermia (TH) as a neuroprotective measure (1). The effects of $\mathrm{TH}$ on cardiac output (CO), systemic vascular resistance (SVR), blood pressure (BP), and cerebral perfusion in this cohort is becoming an area of active research. Several studies have documented impaired myocardial performance (measured conventionally and by speckle tracking echocardiography) and a reduced $\mathrm{CO}$ measured by echocardiography in infants with $\mathrm{NE}$ undergoing $\mathrm{TH}$ when compared to healthy term counterparts $(2,3)$. Animal data suggest that the fall in $\mathrm{CO}$ is secondary to a decreased heart rate and stroke volume (SV), accompanied by a concomitant increase in SVR (4-6). The decrease in metabolic demand associated with $\mathrm{TH}$ is thought to offset the potential detrimental effect of a low blood flow state in those infants. Near-infrared spectroscopy (NIRS) may also play a role in monitoring cerebral perfusion and oxygen extraction during TH. Recent studies have demonstrated good correlation between NIRS-measured cerebral mixed venous saturation values $\left(\mathrm{SctO}_{2}\right)$ and cerebral blood flow measured using magnetic resonance imaging (MRI) in infants undergoing TH (7). In addition, NIRS may have a prognostic role in this population $(8,9)$.

However, studies to date assessing the hemodynamic status of infants undergoing TH have used echocardiography which produces discrete, isolated measurements of function and output. The ability to assess CO (including SV), SVR, BP, and heart rate in addition to $\mathrm{SctO}_{2}$ in a continuous, noninvasive manner would enable a more detailed assessment of the interaction of all the above parameters in the setting of $\mathrm{TH}$ and NE in real time. Transthoracic bioreactance is a new technique of continuous noninvasive cardiac output monitoring (NICOM) that has demonstrated good reliability against invasive measures of $\mathrm{CO}$ in studies involving adults, children, and small animals (10-15). Our group has recently demonstrated the feasibility and reliability of NICOM (NICOM, Cheetah Medical, Portland, OR) compared with echocardiography in stable late-preterm and term neonates, and in premature infants following patent ductus arteriosus (PDA) ligation $(16,17)$. Its application in neonates with NE however remains unexplored.

In this study, we aim to (i) perform continuous measurements of $\mathrm{CO}, \mathrm{SV}, \mathrm{SVR}$, heart rate, $\mathrm{BP}$, and $\mathrm{SctO}_{2}$ using NICOM and NIRS in infants with NE undergoing TH throughout the cooling and rewarming period; and (ii) compare NICOMmeasured $\mathrm{CO}$ with echocardiography-measured $\mathrm{CO}$ (echo-CO) over three time points. We hypothesized that NICOM use in infants with NE is feasible and can provide a reliable method of $\mathrm{CO}$ assessment in this population.

\footnotetext{
'Department of Neonatology, The Rotunda Hospital, Dublin, Ireland; ${ }^{2}$ Department of Radiology, Children's University Hospital, Dublin, Ireland; ${ }^{3}$ Department of Neonatology, Coombe Women and Infants University Hospital, Dublin, Ireland; ${ }^{4}$ Department of Paediatrics, School of Medicine, The Royal College of Surgeons in Ireland, Dublin, Ireland. Correspondence: Afif EL-Khuffash (afifelkhuffash@rcsi.ie) 


\section{Articles $\mid$ Forman et al.}

\section{METHODS}

Study Setting, Patient Population, and Clinical Characteristics This was a prospective observation study carried out in the neonatal intensive care units of the Rotunda Hospital and the Coombe Women and Infants University Hospital, Dublin, Ireland. Ethical approval was obtained from the local Research Ethics Committees and written informed consent was obtained from parents of all participants prior to enrollment. All infants were either passively or actively cooled within six hours following birth. Parents were given up to $12 \mathrm{~h}$ following the initiation of $\mathrm{TH}$ to consider the study. Infants greater than 35 weeks gestation with a diagnosis of NE who underwent $\mathrm{TH}$ were all eligible provided they fulfilled two of the following criteria based on the TOBY study (18): Apgar score of $\leq 5$ at $10 \mathrm{~min}$ after birth; continued need for endotracheal or mask ventilation at $10 \mathrm{~min}$ after birth; acidosis within $60 \mathrm{~min}$ of birth (defined as any occurrence of umbilical cord, arterial, or capillary $\mathrm{pH}$ $<7.00$ or a base deficit $\geq 16 \mathrm{mmol} / \mathrm{l}$ ); and/or clinical seizures or moderate to severe encephalopathy using the Sarnat grading system (19). We excluded infants who were unlikely to survive beyond the cooling period.

All clinical care decisions including sedation and inotrope use were left to the discretion of the clinical team. Clinical characteristics including gestation, birth weight, mode of delivery, Apgar scores, first $\mathrm{pH}$, base excess, and lactate, the need for resuscitation at birth, the use of sedation and inotropes during $\mathrm{TH}$, the presence of seizures, and the use of antiepileptic medication were recorded. The clinical team was blinded to the NICOM and NIRS measurements.

\section{Continuous Hemodynamic Monitoring}

We used the NICOM system (Cheetah Medical Inc, MA) which employs transthoracic bioreactance to obtain continuous hemodynamic readings during TH and rewarming $(16,17)$. Bioreactance is the analysis of the variation in the frequency spectra of a delivered oscillating current when it traverses the thoracic cavity. This is obtained by placing four emitting and receiving electrodes in a manner that "boxes" the heart. Each electrode sensor strip consists of two contact points. Upper thoracic electrode strips were placed over the mid-clavicles and upper back bilaterally. The lower electrode sensors are placed between the sixth and seventh intercostal spaces at the mid-axillary line. NICOM measurements of SV and CO are obtained every minute. SVR is calculated by the NICOM system on an hourly basis as the mean BP is manually entered using the following formula: $\mathrm{SVR}=(\mathrm{BP} \times 80) / \mathrm{CO}$ as per the entered algorithm; where BP is the mean arterial pressure.

NIRS monitoring of cerebral mixed venous saturations was performed using the INVOS Somanetics system (Medtronic, MN) and cerebral oximetry neonatal sensors. The sensors were applied to the skull over the frontal lobes at the center of the forehead and secured using clear bandages for the duration of $\mathrm{TH}$ and rewarming. NIRS provides continuous $\mathrm{SctO}_{2}$ readings using spatially-resolved spectroscopy (20).

\section{MRI Findings}

All enrolled infants underwent a brain MRI on day 5-10 after delivery. A pediatric radiologist who is blinded to NICOM and NIRS results reported and scored the MRI results using the Barkovich criteria (21). This employs a combination five-point score including components of both basal ganglia and watershed patterns of injury. Patients were divided into "normal" $($ score $=0)$ and "abnormal" (score $=1-4)$ neuroimaging groups for the purpose of data analysis.

\section{Echocardiography Measurements}

Echocardiograms were performed using the Vivid S6 machine (GE Medical, Milwaukee, WI) and a $7 \mathrm{MHz}$ cardiac multi-frequency probe on infants recruited from the Rotunda Hospital site. After TH initiation, infants underwent echocardiography assessments of CO on day 1 ( $10 \mathrm{~h}$ of $\mathrm{TH})$, prior to rewarming $(70 \mathrm{~h}$ of $\mathrm{TH})$, and at the end of the rewarming period ( $100 \mathrm{~h}$ after commencement of $\mathrm{TH}$ ). The 10-h time point was chosen to ensure that all the infants undergoing echocardiography also had NICOM monitoring. The delay in applying NICOM monitoring was to facilitate parental informed consent. The 70-h time point was chosen as the time of maximal cooling duration just prior to rewarming. The 100 -h time point represented the completion of rewarming. At the time of the first echocardiogram, the NICOM and echocardiography internal clocks were synchronized. All echocardiograms were performed and analyzed by one investigator (CRB) to avoid inter-rater variability. The investigator was blinded to the NICOM readings during the echocardiogram. The echo-CO was calculated based on previously described methodology (22). At a later date, complete NICOM measurements for each infant were downloaded from the NICOM machine. The NICOM-CO measurements that exactly corresponded to the timed minute of acquisition of the echocardiography data were identified. These NICOM and echocardiography data were compared.

\section{Statistical Analysis}

Continuous data were presented as median (interquartile range) or as means (SD) as appropriate. Categorical data were presented as absolute numbers and proportions. Two group analysis was conducted using the Mann-Whitney $U$ test for continuous variables or the $\chi^{2}$ test for categorical variables. One-way repeated measures ANOVA was used to assess the change in the hemodynamic measurements with respect to time. Pairwise comparison carried out

Table 1. Hemodynamic measurements at three time points

\begin{tabular}{|c|c|c|c|c|}
\hline & During cooling $(10 \mathrm{~h})$ & Pre-rewarming $(70 \mathrm{~h})$ & Post-rewarming $(100 \mathrm{~h})$ & ANOVA $P$ \\
\hline Temperature $\left(^{\circ}\right)$ & $33.4(0.4)$ & $33.5(0.1)$ & $36.4(0.8)^{a}$ & $<0.001$ \\
\hline NICOM-CO (ml/kg/min) & $82(31)$ & $83(38)$ & $120(57)^{\mathrm{a}}$ & 0.003 \\
\hline NICOM/Echo percentage bias (\%) & $21(14)$ & $29(10)$ & $32(9)$ & 0.14 \\
\hline Cerebral regional $\mathrm{O} 2$ saturation (\%) & $80(9)$ & $85(6)^{a}$ & $85(5)^{\mathrm{a}}$ & 0.005 \\
\hline Mean BP $(\mathrm{mmHg})$ & $45(5)$ & $50(6)$ & $50(5)^{a}$ & 0.002 \\
\hline Heart rate & $102(13)$ & $97(15)$ & $116(16)^{\mathrm{a}}$ & $<0.001$ \\
\hline
\end{tabular}

ANOVA, analysis of variance; Echo-CO, echocardiography-derived cardiac output; Mean BP, mean blood pressure; NICOM-CO, NICOM-derived cardiac output; SV, stroke volume; SVR, systemic vascular resistance.

Values are presented as means (SD) or medians (IQR). One was ANOVA with repeated measures was used to assess change over time.

${ }^{a} P$ value $<0.05$ compared to baseline (Bonferroni adjustment). 
a

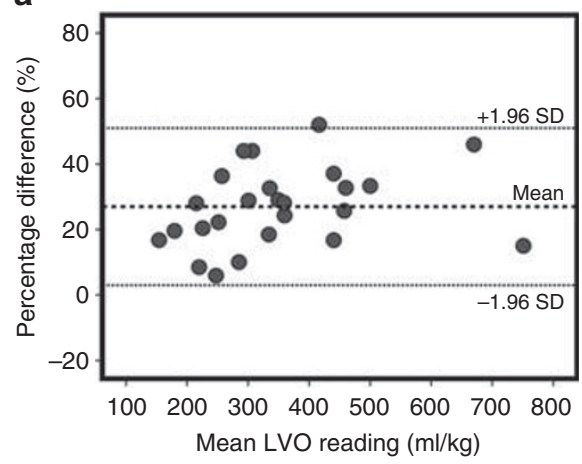

\section{b}

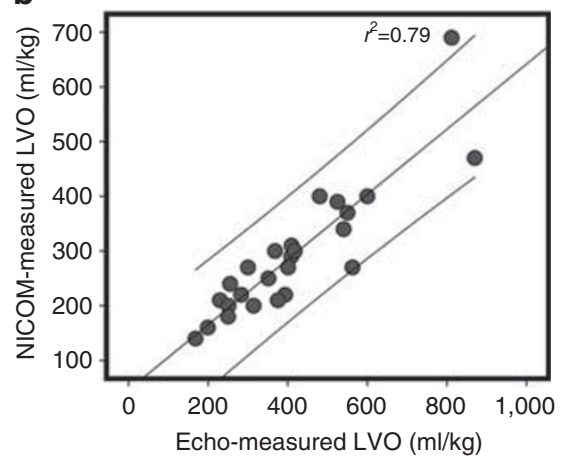

Figure 1. Bland-Altman Analysis (a) and Correlation (b) between echocardiography and noninvasive cardiac output monitoring (NICOM)-measured cardiac output. NICOM and Echo cardiac output (depicted as left ventricular output, LVO) was not indexed to weight for graphical representation.
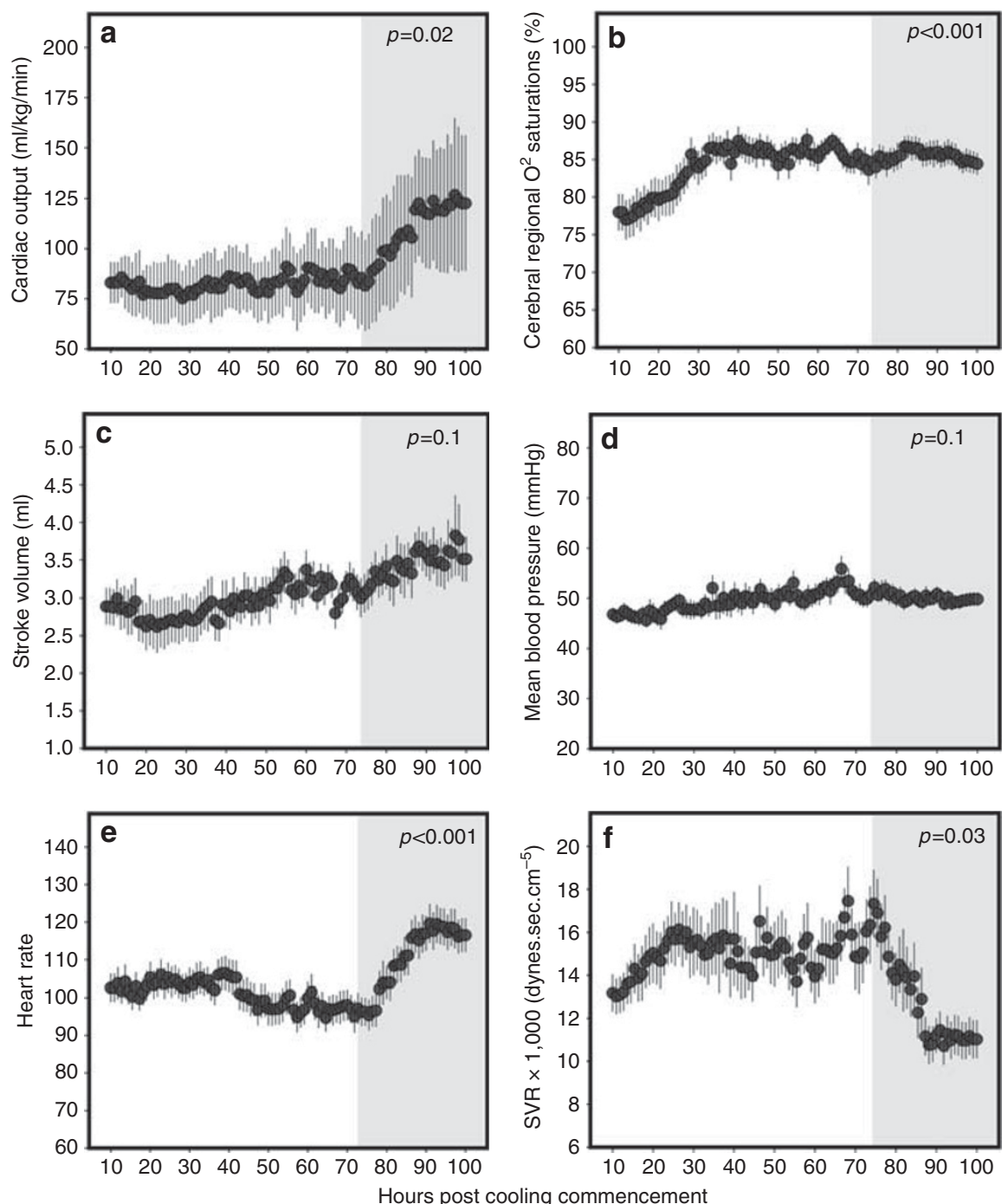

Figure 2. Continuous hemodynamic measurements in the cohort over the study period. Changes in cardiac output (a), cerebral regional oxygen saturation (b), stroke volume (c), mean blood pressure (d), heart rate (e), and systemic vascular resistance (f) are presented. The white area represents the hypothermia phase and the gray area represents the rewarming phase. Values are presented as means (dark gray circles) and the black lines represent the standard error. One-way ANOVA with repeated measures was used to assess the change over time. SVR, systemic vascular resistance. 
Table 2. Clinical characteristics of infants with and without an abnormal MRI

\begin{tabular}{lccc}
\hline & $\begin{array}{c}\text { Normal MRI } \\
(n=10)\end{array}$ & $\begin{array}{c}\text { Abnormal MRI } \\
(n=10)\end{array}$ & $P$ \\
\hline Gestation (weeks) & $38.7(38.0-40.1)$ & $38.9(38.0-39.1)$ & 0.42 \\
Birth weight (kg) & $3.53(3.32-3.90)$ & $3.34(2.50-4.23)$ & 0.83 \\
Cesarean section & $8(80)$ & $5(50)$ & 0.35 \\
Male gender & $8(80)$ & $4(40)$ & 0.17 \\
Outborn & $5(50)$ & $7(70)$ & 0.65 \\
Apgar Scores & & & \\
$\quad 1$ min & $0(0-1)$ & $3(3-5)$ & $<0.01$ \\
$\quad 5$ min & $5(1-5)$ & $4(2-7)$ & 0.19 \\
$\quad 10$ min & $5(3-9)$ & $7(5-9)$ & 0.76 \\
Lowest first-hour pH & $6.96(6.89-7.12)$ & $6.90(6.82-7.01)$ & 0.74 \\
Base excess & -15.8 & -14.8 & 0.58 \\
& $(-20.4--12.3)$ & $(-19.8--12.3)$ & \\
First lactate (mmol/l) & $12.8(10.4-14.4)$ & $10.9(9.5-16.6)$ & 0.91 \\
Inotropes & $5(50)$ & $4(40)$ & 1.0 \\
Seizure activity & $1(10)$ & $4(40)$ & 0.30 \\
\hline
\end{tabular}

MRI, magnetic resonance imaging.

Data are presented as medians (interquartile range) or as count (\%).

between two time points (70 and $100 \mathrm{~h}$ ) and baseline $(10 \mathrm{~h})$ was conducted using the Bonferroni adjustment. Two-way repeated measures ANOVA was used to assess the difference in the hemodynamic measurements between two groups (normal vs. abnormal MRI) over time.

A systematic bias of $31 \pm 8 \%$ between the two methods of $\mathrm{CO}$ assessment was demonstrated previously, with NICOM underreading the echocardiography values (17). Therefore, differences in measurement between the two methods were expressed as a percentage ((echo-CO-NICOM-CO)/echo-CO). We compared the two methods using the Bland-Altman analysis, and assessed the correlation between the two methods using Pearson's correlation coefficient. A $P$ value $<0.05$ was considered significant. SPSS (IBM House, Dublin, Ireland) (IBM version 22) was used to perform the statistical analysis.

\section{RESULTS}

Over the study period, 27 infants were eligible for inclusion. Three were excluded due to the unavailability of the NICOM monitor, two were unlikely to survive (and subsequently had care redirected to palliation) and two families refused consent. Twenty infants with a median (IQR) gestation of 40.0 (38.741.1) weeks and a median birth weight of $3.6(3.4-4.0) \mathrm{kg}$ were enrolled over an eighteen-month period. Thirteen (65\%) were delivered by cesarean section and twelve (60\%) were male. Ten (50\%) infants were outborn. Their 1, 5, and $10 \mathrm{~min}$ Apgar scores were 2 (0-4), 5 (2-7], and 6 (4-9) respectively. The cohort had an admission $\mathrm{pH}$ of 6.93 (6.85-7.07), a base excess of $-14.9(-19.3--11.8)$, and an initial arterial lactate of $12.8(9.0-14.8) \mathrm{mmol} / \mathrm{l}$. TH was commenced within $6 \mathrm{~h}$ following birth in all infants. All infants were in receipt of either morphine $(n=11)$, fentanyl $(n=11)$ or midazolam $(n=5)$ for sedation during TH. NICOM hemodynamic measurements (CO, SVR, BP, SV, and HR) and NIRS measurements $\left(\mathrm{SctO}_{2}\right)$ were all commenced within $10 \mathrm{~h}$ of
$\mathrm{TH}$ initiation. During the cooling period, all infants were maintained at a temperature between $33^{\circ}$ to $34^{\circ}$ centigrade and were rewarmed following $72 \mathrm{~h}$ of $\mathrm{TH}$ over an $18 \mathrm{~h}$ period (Table 1).

NICOM and NIRS assessments were feasible in all enrolled infants. Eight infants underwent echocardiography assessments of $\mathrm{CO}$ over three time points. NICOM-CO were lower than echo-CO with a bias of $27 \%$ (limits of agreement $8-51 \%$ ). There was a strong correlation between echo-CO and NICOM-CO $\left(r^{2}=0.79, P<0.001\right)$ (Figure 1). The bias between the two methods remained constant throughout the study period with no difference noted between the cooling and rewarming phases. There was an increase in both NICOM-CO and echo-CO following the rewarming period (Table 1).

$\mathrm{CO}$ was lower during TH and significantly increased during rewarming. $\mathrm{SctO}_{2}$ significantly increased over the first $30 \mathrm{~h}$ of $\mathrm{TH}$ and stayed higher for the remainder of the study period (Table 1, Figure 2). There was a significant rise in NICOMmeasured SVR over the first $30 \mathrm{~h}$ of TH. SVR subsequently decreased during rewarming. There was no change in mean arterial BP or SV over the study period. Heart rate significantly increased during rewarming (Table 1, Figure 2).

Nine infants received inotropes during the study period. There was no difference in gestation, birth weight, Apgar scores, $\mathrm{pH}$, base excess, or lactate between infants in receipt of inotropes and those without (data not shown). All infants received dopamine, with two infants receiving adrenaline and one infant receiving dobutamine as adjuvant therapy. The median time of commencement was $6(2-18) \mathrm{h}$, with a total duration of $49(23-83) \mathrm{h}$. The median BP at inotrope commencement was $44(42-47)$. There was no significant change in any of the measurements in the $24 \mathrm{~h}$ period following the commencement of inotropes (data not shown).

All infants underwent a brain MRI between days 5-10 of age. Ten infants had an abnormal MRI. Table 2 summarizes the differences in characteristics between infants with and without a normal MRI. There were no significant differences between the two groups with the exception of the 1 min Apgar score which was higher in the abnormal MRI group (Table 2). Figure 3 illustrates the hemodynamic profiles in the two groups. Infants in the abnormal MRI group had lower CO, and SV and a higher SVR during TH. However those differences did not reach statistical significance (Figure 3 ).

\section{DISCUSSION}

In this prospective study, we applied a relatively novel method of continuous hemodynamic assessment (NICOM) to infants with NE throughout the cooling and rewarming periods and compared $\mathrm{CO}$ measured using this method with that of echocardiography. The use of NICOM and NIRS in this population is feasible as measurements were obtainable in all recruited infants. We did not encounter difficulty in applying the sensors on the infants or maintaining the sensors in place throughout the study period. In addition, those sensors did not impede routine clinical monitoring or care provision to those infants. 


\section{Hemodynamics in neonatal encephalopathy Articles}
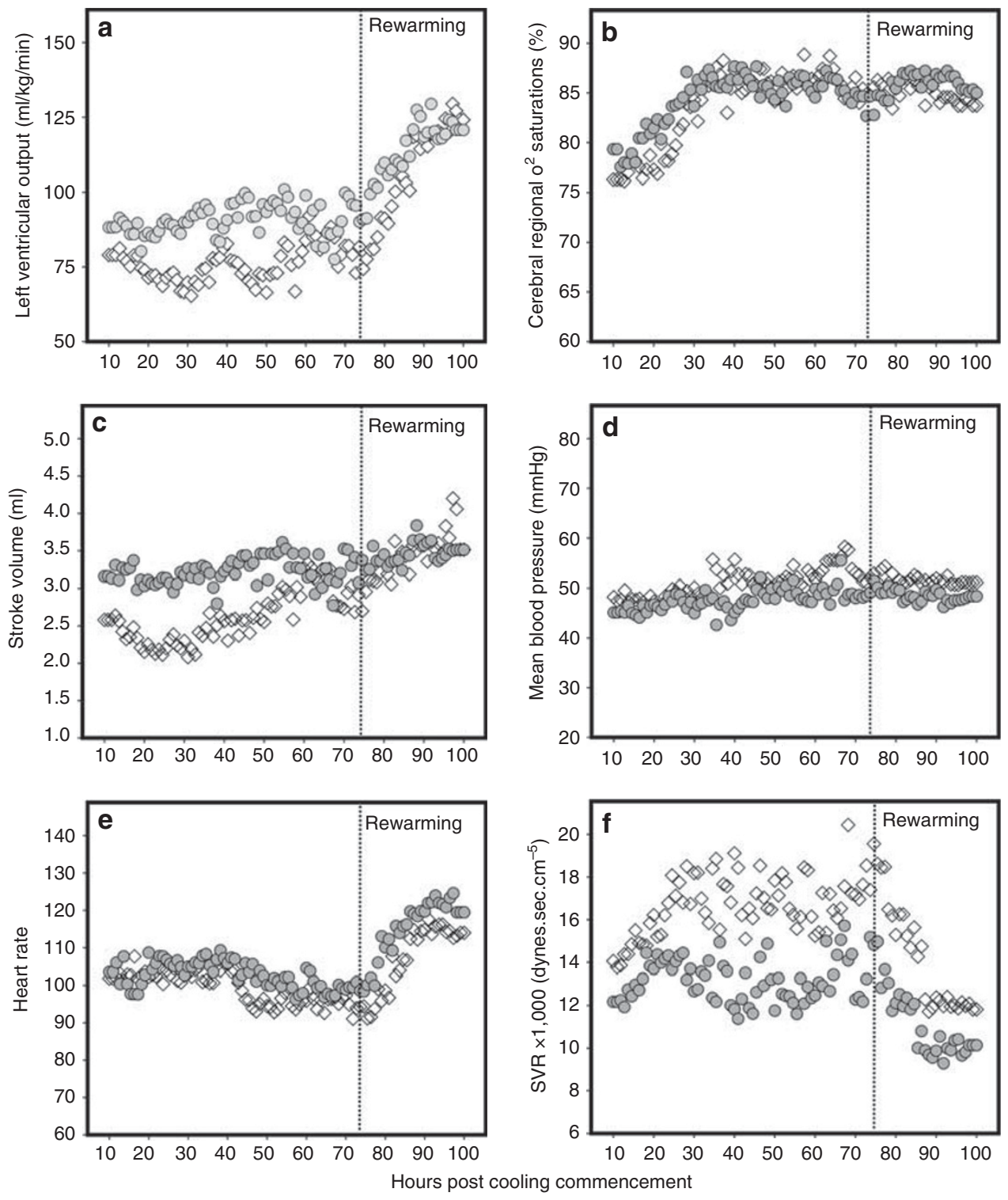

Figure 3. Change in the hemodynamic profile in infants with (white diamonds) and without (gray circles) an abnormal magnetic resonance imaging (MRI). Changes in cardiac output (a), cerebral regional oxygen saturation (b), stroke volume (c), mean blood pressure (d), heart rate (e), and systemic vascular resistance (f) are presented. One-way ANOVA with repeated measures was used to assess the change over time. There was no significant change in any of those measurements between the groups over time (all ANOVA $P>0.05$ ). SVR, systemic vascular resistance.

We illustrated that NICOM under-reads echo-CO by an average of $27 \%$; This systematic bias is similar to the bias illustrated by our group in two other patient populations: healthy term and late-preterm infants, and preterm infants following patent ductus arteriosus ligation $(16,17)$. While acknowledging that neither method can be regarded as a gold-standard, we have previously provided a rationale for this systematic bias between the two methods (17). The NICOM algorithm used to estimate aortic diameter size in the neonatal population is extrapolated from adults which may have resulted in the lower NICOM values of CO (23). Conversely, echocardiography may overestimate $\mathrm{CO}$ readings as it uses the velocity of blood flowing through the central portion of the aortic root to estimate $\mathrm{CO}$. This may significantly overestimate the true overall velocity of blood flowing through the aortic root as the higher velocity is found in the center of the vessel while flow along the periphery (not measured by echocardiography) is significantly lower (24). We also found that the bias appears to be constant throughout the cooling and rewarming phases with no significant differences between the three NICOM and echocardiography measurement time points. This has important implications as it suggests that body temperature changes do not appear to affect the bioreactance signal properties. Animal studies support these findings. In a study of nine openchest pigs, where blood flow was controlled with cardiopulmonary bypass, NICOM-CO measurement correlated well with cardiopulmonary bypass measurements $(r=0.84)$ across two blood temperatures $\left(36^{\circ}\right.$ and $\left.38^{\circ} \mathrm{C}\right)$. Although this temperature 


\section{Articles $\mid$ Forman et al.}

range is different to that in our study, the data suggest the relative lack of effect temperature has on NICOM-CO measurement (23).

We illustrated that NICOM monitoring of infants with NE during $\mathrm{TH}$ and rewarming reflects expected hemodynamic changes during this process, adding further support to its reliability in this patient cohort. Left ventricular output is lower during hypothermia and increased during rewarming. This change appears to be driven by changes in heart rate which mirrored the increased CO rather than SV, which increased at a lower rate. There was a rise in SVR during cooling which decreased with rewarming. There was no change in mean BP during the study period. Hypothermia is associated with a reduction in metabolic demand and a resultant fall in $\mathrm{CO}$ (25). Adult human data and animal models of TH have previously demonstrated this rise in SVR following the lowering of body temperature $(26,27)$. The rise in SVR may be a contributing mechanism to the maintenance of cerebral perfusion in the cooled neonatal population (28). The early rise in $\mathrm{SctO}_{2}$ seen in our cohort occurring in conjunction with the rising SVR supports this theory. $\mathrm{SctO}_{2}$ remained elevated during rewarming despite a falling SVR. The increase in CO may have maintained cerebral perfusion.

We found that infants with evidence of brain injury seen on MRI had a higher SVR and a lower CO driven by a lower SV rather than heart rate (although none of those differences reached statistical significance, likely due to the small number of subjects). This finding suggests that the more severely affected infants may also have myocardial injury, with an inability to maintain adequate SV in the face of increasing afterload. This relationship warrants further study in a larger cohort to confirm those findings.

The effect of inotropes on the cardiovascular system during $\mathrm{TH}$ warrants further study. We found no demonstrable change in CO, mean BP or SVR following the introduction of inotropes in this population. The lack of change may highlight the lack of response to inotropes during $\mathrm{TH}$ (26), however, no meaningful conclusions can be drawn from this small sample size and the relatively diverse number of inotropes used in this study.

This study has several limitations: the relative small number of infants may have resulted in missing important differences in the measures between infants with and without brain injury. In addition, the relative delay in applying NICOM (done to facilitate obtaining consent) may have resulted in missing important hemodynamic changes during the early cooling phase. Although NICOM has been validated against echocardiography in neonates $(16,17)$, further work is required to assess its precision and its ability to detect important changes over time. Our study was not powered to assess the effect of other important factors such as sedation, inotrope use, and the presence of seizures on the hemodynamic status of those infants.

\section{CONCLUSION}

We have demonstrated that the assessment of the hemodynamic status of infants with $\mathrm{NE}$ undergoing $\mathrm{TH}$ in addition to cerebral perfusion in a continuous fashion is feasible and reflects the expected hemodynamic changes occurring as a result of $\mathrm{TH}$ and rewarming in infants with NE. Further studies are required to assess NICOM's clinical applicability in this condition in a larger cohort.

\section{STATEMENT OF FINANCIAL SUPPORT}

This study was funded by multiple sources: EU FP7/2007-2013 grant (agreement no. 260777, The HIP Trial); the Friends of the Rotunda Research Grant (Reference: FoR/EQUIPMENT/101572); Health Research Board Mother and Baby Clinical Trials Network Ireland (CTN-2014-10); Medical Research Charities Group/Health Research Board/Friends of the Rotunda Research Grant (HANDLE Study, MRCG-2013-9).

Disclosure: The authors declare no conflict of interest.

\section{REFERENCES}

1. Jacobs SE, Berg M, Hunt R, Tarnow-Mordi WO, Inder TE, Davis PG. Cooling for newborns with hypoxic ischaemic encephalopathy. Cochrane Database Syst Rev 2013;1:CD003311.

2. Costa S, Zecca E, De RG, et al. Is serum troponin T a useful marker of myocardial damage in newborn infants with perinatal asphyxia? Acta Paediatr 2007;96:181-4.

3. Czernik C, Rhode S, Helfer S, Schmalisch G, Buhrer C. Left ventricular longitudinal strain and strain rate measured by $2-\mathrm{D}$ speckle tracking echocardiography in neonates during whole-body hypothermia. Ultrasound Med Biol 2013;39:1343-9.

4. Fugelseth D, Satas S, Steen PA, Thoresen M. Cardiac output, pulmonary artery pressure, and patent ductus arteriosus during therapeutic cooling after global hypoxia-ischaemia. Arch Dis Child Fetal Neonatal Ed 2003;88:F223-8.

5. Gebauer CM, Knuepfer M, Robel-Tillig E, Pulzer F, Vogtmann C. Hemodynamics among neonates with hypoxic-ischemic encephalopathy during whole-body hypothermia and passive rewarming. Pediatrics 2006;117:843-50.

6. Dudgeon DL, Randall PA, Hill RB, McAfee JG. Mild hypothermia: its effect on cardiac output and regional perfusion in the neonatal piglet. J Pediatr Surg 1980;15:805-10.

7. Wintermark P, Hansen A, Warfield SK, Dukhovny D, Soul JS. Nearinfrared spectroscopy versus magnetic resonance imaging to study brain perfusion in newborns with hypoxic-ischemic encephalopathy treated with hypothermia. Neuroimage 2014;85(Pt 1): 287-93.

8. Ancora G, Maranella E, Grandi S, et al. Early predictors of short term neurodevelopmental outcome in asphyxiated cooled infants. A combined brain amplitude integrated electroencephalography and near infrared spectroscopy study. Brain Dev 2013;35:26-31.

9. Massaro AN, Govindan RB, Vezina G, et al. Impaired cerebral autoregulation and brain injury in newborns with hypoxic-ischemic encephalopathy treated with hypothermia. J Neurophysiol 2015;114: 818-24.

10. Heerdt PM, Wagner CL, DeMais M, Savarese JJ. Noninvasive cardiac output monitoring with bioreactance as an alternative to invasive instrumentation for preclinical drug evaluation in beagles. J Pharmacol Toxicol Methods 2011;64:111-8.

11. Marik PE, Levitov A, Young A, Andrews L. The use of bioreactance and carotid Doppler to determine volume responsiveness and blood flow redistribution following passive leg raising in hemodynamically unstable patients. Chest 2013;143:364-70.

12. Marque S, Cariou A, Chiche JD, Squara P. Comparison between FlotracVigileo and Bioreactance, a totally noninvasive method for cardiac output monitoring. Crit Care 2009;13:R73.

13. Raval NY, Squara P, Cleman M, Yalamanchili K, Winklmaier M, Burkhoff D. Multicenter evaluation of noninvasive cardiac output measurement by bioreactance technique. J Clin Monit Comput 2008;22: 113-9. 
14. Squara P, Denjean D, Estagnasie P, Brusset A, Dib JC, Dubois C. Noninvasive cardiac output monitoring (NICOM): a clinical validation. Intensive Care Med 2007;33:1191-4.

15. Vandormael Y, Vu T, GeWillig M, Vlasselaers D. Non-invasive cardiac output monitoring in children: clinical validation. Crit Care 2010;14 (Suppl 1): P112.

16. Weisz DE, Jain A, Ting J, McNamara PJ, El-Khuffash A. Non-invasive cardiac output monitoring in preterm infants undergoing patent ductus arteriosus ligation: a comparison with echocardiography. Neonatology 2014;106:330-6.

17. Weisz DE, Jain A, McNamara PJ, El-Khuffash A. Non-invasive cardiac output monitoring in neonates using bioreactance: a comparison with echocardiography. Neonatology 2012;102:61-7.

18. Azzopardi D, Brocklehurst P, Edwards D, et al. The TOBY Study. Whole body hypothermia for the treatment of perinatal asphyxial encephalopathy: a randomised controlled trial. BMC Pediatr 2008;8:17.

19. Sarnat HB, Sarnat MS. Neonatal encephalopathy following fetal distress. A clinical and electroencephalographic study. Arch Neurol 1976;33: 696-705.

20. Lemmers PM, Toet MC, van BF. Impact of patent ductus arteriosus and subsequent therapy with indomethacin on cerebral oxygenation in preterm infants. Pediatrics 2008;121:142-7.
21. Barkovich AJ, Maroldo TV. Magnetic resonance imaging of normal and abnormal brain development. Top Magn Reson Imaging 1993;5:96-122.

22. El-Khuffash AF, McNamara PJ. Neonatologist-performed functional echocardiography in the neonatal intensive care unit. Semin Fetal Neonatal Med 2011;16:50-60.

23. Keren H, Burkhoff D, Squara P. Evaluation of a noninvasive continuous cardiac output monitoring system based on thoracic bioreactance. Am J Physiol Heart Circ Physiol 2007;293:H583-9.

24. Zaazaa AA, Fathy I, Ayman O, Hassanin A, Ghanem H. A pilot study of penile hemodynamics in men with penile curvatures. Int J Impot Res 2017;29:86-8.

25. Espinoza A, Kerans V, Opdahl A, et al. Effects of therapeutic hypothermia on left ventricular function assessed by ultrasound imaging. J Am Soc Echocardiogr 2013;26:1353-63.

26. Wood T, Thoresen M. Physiological responses to hypothermia. Semin Fetal Neonatal Med 2015;20:87-96.

27. Demirgan S, Erkalp K, Sevdi MS, et al. Cardiac condition during cooling and rewarming periods of therapeutic hypothermia after cardiopulmonary resuscitation. BMC Anesthesiol 2014;14:78.

28. Hochwald O, Jabr M, Osiovich H, Miller SP, McNamara PJ, Lavoie PM. Preferential cephalic redistribution of left ventricular cardiac output during therapeutic hypothermia for perinatal hypoxic-ischemic encephalopathy. J Pediatr 2014;164:999-1004 el. 